\title{
Utility of Repeat Head CT in Patients with Blunt Traumatic Brain Injury Presenting with Small Isolated Falcine or Tentorial Subdural Hematomas
}

\author{
(D).K. Devulapalli, (D).F. Talbott, (D). Narvid, (D)A. Gean, (D) Behani, (D) G. Manley, (D)A. Uzelac, (D)E. Yuh, and (D).C. Huang
}

\begin{abstract}
BACKGROUND AND PURPOSE: In blunt traumatic brain injury with isolated falcotentorial subdural hematoma not amenable to neurosurgical intervention, the routinely performed, nonvalidated practice of serial head CT scans frequently necessitates increased hospital resources and exposure to ionizing radiation. The study goal was to evaluate clinical and imaging features of isolated falcotentorial subdural hematoma at presentation and short-term follow-up.
\end{abstract}

MATERIALS AND METHODS: We performed a retrospective analysis of patients presenting to a level 1 trauma center from January 2013 to March 2015 undergoing initial and short-term follow-up CT with initial findings positive for isolated subdural hematoma along the falx and/or tentorium. Patients with penetrating trauma, other sites of intracranial hemorrhage, or depressed skull fractures were excluded. Patient sex, age, Glasgow Coma Scale score, and anticoagulation history were obtained through review of the electronic medical records.

RESULTS: Eighty patients met the inclusion criteria ( 53 males; 27 females; median age, 61 years). Of subdural hematomas, $57.1 \%$ were falcine, $33.8 \%$ were tentorial, and $9.1 \%$ were mixed. The mean initial Glasgow Coma Scale score was 14.2 (range, 6-15). Isolated falcotentorial subdural hematomas were small (mean, $2.8 \mathrm{~mm}$; range, 1-8 mm) without mass effect and significant change on follow-up CT (mean, $2.7 \mathrm{~mm}$; range, $0-8 \mathrm{~mm} ; P=.06$ ), with an average follow-up time of 10.3 hours (range, 3.9-192 hours). All repeat CTs demonstrated no change or decreased size of the initial subdural hematoma. No new intracranial hemorrhages were seen on follow-up CT.

CONCLUSIONS: Isolated falcotentorial subdural hematomas in blunt traumatic brain injury average $2.8 \mathrm{~mm}$ in thickness and do not increase in size on short-term follow-up CT. Present data suggest that repeat CT in patients with mild traumatic brain injury with isolated falcotentorial subdural hematoma may not be necessary.

ABBREVIATIONS: GCS = Glasgow Coma Scale; $\mathrm{SDH}=$ subdural hematoma; $\mathrm{TBI}=$ traumatic brain injury

$\mathrm{T}$ raumatic brain injury (TBI) is a common neurologic injury affecting 1.7 million Americans each year. ${ }^{1}$ Up to $30 \%$ of all patients with TBI develop acute subdural hematomas (SDHs), with a higher prevalence in those patients sustaining more severe injuries. ${ }^{2-4}$ While the relative distribution of SDH by location has not been well-studied, 1 retrospective series of 646 patients found $73.9 \%$ of SDHs located along the cerebral convexity, whereas $14 \%$, and $11 \%$ were falcine or tentorial, respectively. ${ }^{5}$ Convexity

Received May 4, 2017; accepted after revision November 30

From the Department of Radiology and Biomedical Imaging (K.K.D., J.F.T., J.N., A.G., B.R., A.U., E.Y.), University of California, San Francisco and Zuckerberg San Francisco General Hospital, San Francisco, California; Department of Neurological Surgery (G.M., M.C.H.), Zuckerberg San Francisco General Hospital, San Francisco, California; and Department of Neurological Surgery (J.F.T., G.M., E.Y., M.C.H.), Brain and Spinal Injury Center, San Francisco, California.

Please address correspondence to Jason Talbott, MD, PhD, Department of Radiology and Biomedical Imaging, University of California, San Francisco, 400 Parnassus Ave, San Francisco, CA 94110; e-mail: Jason.talbott@ucsf.edu

http://dx.doi.org/10.3174/ajnr.A5557
$\mathrm{SDH}$ is often accompanied by other intracranial injuries and more frequently requires operative intervention. ${ }^{6,7}$ Little, however, has been reported regarding the natural history of isolated falcine and tentorial SDH. Several reported studies suggest that these categorizations of SDH tend to occur in isolation with mild TBI and can be managed nonoperatively., 5,8

Noncontrast CT remains an essential imaging technique in the assessment of TBI, given its sensitivity in the detection of acute intracranial hemorrhage. Many patients experiencing convexity SDH in the setting of TBI undergo initial CT in addition to repeat evaluations. In the absence of specific guidelines and data on falcine and tentorial SDH, these patients also often undergo repeat noncontrast head CT evaluation. Given the paucity of data on falcine and tentorial SDH in TBI, the purpose of this study was to evaluate clinical and imaging features of isolated falcine and tentorial SDH at presentation and short-term follow-up. We hypothesized that in this patient population, repeat imaging demon- 
strates no significant change from baseline and may be of little if any clinical utility.

\section{MATERIALS AND METHODS}

A retrospective analysis of the PACS data base of adult patients presenting to a level 1 trauma center between January 2013 and March 2015 who underwent CT within 24 hours of presentation to the emergency department was performed. Imaging reports were queried with 4 separate searches using the words "subdural hematoma" in conjunction with the terms "falx," "falcine," "tentorium," and "tentorial." Patients with other sites of intracranial hemorrhage, brain contusion, or depressed skull fractures were excluded. Additionally, patients were excluded if there was no history of TBI. Finally, patients were excluded if a repeat CT scan was not performed within 7 days of emergency department presentation to exclude potential confounding repeat trauma. Clinical information, including sex, age, and history of anticoagulation; Glasgow Coma Scale (GCS) score at presentation and discharge; international normalized ratio; platelet count; and admission level of care was obtained through a review of electronic medical records. Mild TBI was defined as a GCS score of 13-15 on presentation. The Zuckerberg San Francisco General Hospital institutional review board approved this study with a waiver of informed consent.

All imaging was assessed by reviewers K.K.D. (fourth-year radiology resident) and J.F.T. (subspecialty trained and boardcertified neuroradiologist with 4 years of attending experience). The thickest dimension of the SDH measured perpendicular to the plane of the tentorium and/or falx on initial and repeat head CT as evaluated in the axial, sagittal, and coronal planes was measured. Hemorrhage location was categorized as falcine, tentorial, or falcotentorial on the basis of location on the initial CT scan. (Figs 1 and 2).

Outcome measures included the number of patients with an increase in size of the SDH on repeat imaging and the difference in mean SDH size between the initial and repeat scans. Descriptive statistics of continuous and categoric variables in addition to comparative analysis of means using a paired sample $t$ test were performed using JASP, Version 0.8.0.1 (www.jasp-stats.org). $P<$ .05 was considered statistically significant.

\section{RESULTS}

According to an internal Department of Neurosurgery data base maintained at our institution, the neurosurgery service evaluated 1798 cases of TBI. Of these, 1414 had a GCS score of $\geq 13$, one hundred forty-five had a GCS score of 9-12, and 239 had a GCS score of $\leq 8$. Of 98 patients identified in our PACS query with imaging reports of falcine and/or tentorial subdural hematomas, 80 adult patients were identified as having isolated falcine or tentorial SDH with repeat imaging performed within 7 days of the initial presentation. Eleven patients were excluded due to lack of follow-up imaging, 4 patients were excluded because of multicompartmental intracranial hemorrhage, 2 patients were excluded on the basis of equivocal findings for hemorrhage on initial CT, and 1 patient was excluded secondary to an equivocal history of TBI.

Demographic and clinical data are presented in Table 1. The

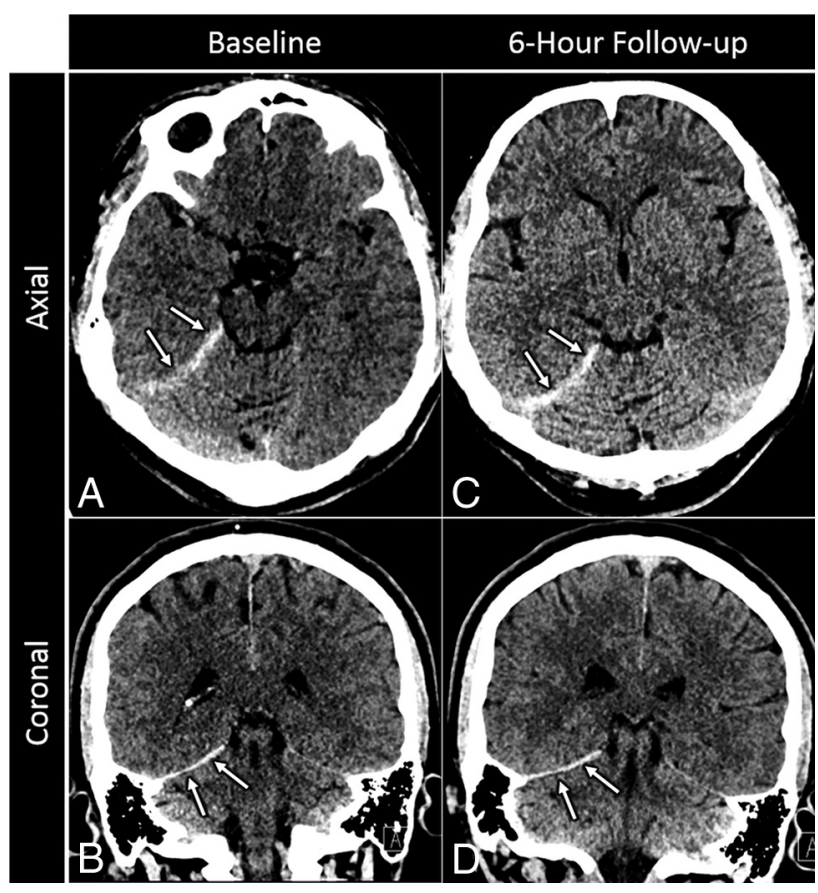

FIG 1. Sample isolated tentorial SDH. A 78-year-old man who presented after a fall. Baseline axial $(A)$ and coronal $(B)$ images from a noncontrast head CT reveal a small, 3-mm-thick acute subdural hematoma along the right tentorium (arrows) without associated mass effect. Axial $(C)$ and coronal $(D)$ images from a noncontrast follow-up CT performed 6 hours later reveal no new hemorrhage or interval change in size of the initial tentorial SDH (arrows).

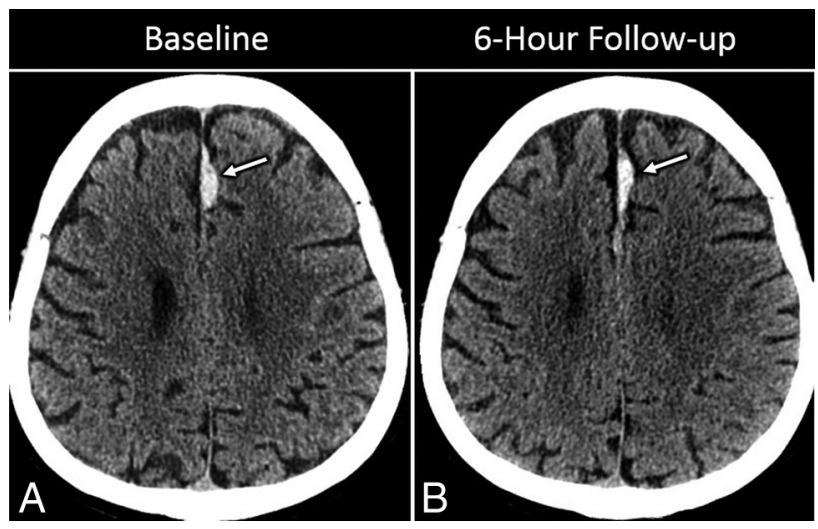

FIG 2. Sample isolated falcine SDH. A 65-year-old man with headache after a fall. Axial image ( $A$ ) from an initial noncontrast head $C T$ shows a lobulated and relatively focal acute high-density SDH along the left side of the anterior falx (arrow). On follow-up CT (B), there has been some mild local redistribution of acute SDH without significant interval change in the overall size or maximal thickness of the hematoma (arrow).

median age was 61.0 years, with a male predominance $(66.3 \%)$. Seventy-four patients (92.5\%) presented with an initial GCS score of 13-15, with an initial mean GCS score of 14.2 (range, 6-15). The mean discharge GCS score was 14.9 (range, 13-15). Overall, 69 patients $(86.3 \%)$ were admitted to the hospital for SDH management. All patients were managed nonoperatively.

Six patients $(7.5 \%)$ were anticoagulated, and an additional 10 $(12.5 \%)$ were on antiplatelet therapy. The average international normalized ratio for anticoagulated patients was $2.9 \pm 1.1$ (range, $1.5-4.9)$.

AJNR Am J Neuroradiol 39:654-57 Apr 2018 www.ajnr.org 655 
Table 1: Patient characteristics

\begin{tabular}{lc}
\multicolumn{1}{c}{ Variable } \\
\hline Age (median) (range) (yr) & 61 (18-97) \\
Male/female & $53: 27$ \\
INR (mean) (range) & $1.18(0.8-4.9)$ \\
$\quad$ All patients with INR within 24 hr of & \\
$\quad$ injury ( $n=78)$ & $2.92(1.5-4.9)$ \\
All anticoagulated patients ( $n=6)$ & $234.2(83-437)$ \\
Platelet count (mean) (range) & $14.2(6-15.0)$ \\
Initial GCS score (mean) (range) & 74 \\
GCS 13-15 & 6 \\
GCS $\leq 12$ & \\
Anticoagulation (No.) & 5 \\
$\quad$ Warfarin (Coumadin) & 1 \\
Rivaroxaban & \\
Antiplatelet therapy (No.) & 7 \\
Aspirin & 3 \\
Clopidogrel & \\
Disposition (No.) & 11 \\
Discharged & 36 \\
Neurologic ICU & 33 \\
Other service &
\end{tabular}

Note:-INR indicates international normalized ratio; ICU, intensive care unit.

Table 2: Imaging data

\begin{tabular}{lc}
\multicolumn{1}{c}{ Variable } & No. \\
\hline SDH location & \\
$\quad$ Falcine & $47(57.1 \%)$ \\
Tentorial & $26(33.8 \%)$ \\
$\quad$ Falcotentorial & $7(9.1 \%)$ \\
Time interval from initial to repeat CT (mean) & $10.3(3.9-192)$ \\
$\quad$ (range) (hr) & \\
SDH expansion & 0 \\
$\quad$ Yes & 80 \\
$\quad$ No & \\
SDH size (mean) (range) (mm) & \\
Initial & $2.8 \pm 1.6(1-8)$ \\
Follow-up & $2.7 \pm 1.6(0-8)$ \\
\hline
\end{tabular}

The average time between the initial and repeat examinations was 10.3 hours (Table 2). All 80 patients (100\%) experienced either no change or a decrease in size of the SDH on repeat imaging. There was no statistically significant difference in average SDH size between the initial and follow-up imaging (2.7 versus $1.6 \mathrm{~mm}$, $P=.17$ ). Subgroup analysis of 69 patients with mild TBI also did not yield any statistically significant difference in the mean SDH size between initial and follow-up imaging (2.5 versus $2.4 \mathrm{~mm}$, $P=.12$ ). No significant difference was noted between the mean SDH size between initial and follow-up imaging in 6 patients with a GCS score of $<13$ (2.5 versus $2.5 \mathrm{~mm}$ ).

A single patient with mild TBI experienced clinical deterioration after initial head CT related to polytrauma. This 77-year-old woman was admitted following pedestrian-versus-automobile injury with a GCS score of 14 in the field and 15 on the initial emergency department assessment. The initial head CT showed a 4-mm isolated falcine SDH without additional acute findings. This patient also had multiple rib fractures and became acutely hypotensive (blood pressure equal to 84 systolic and 51 diastolic) in the emergency department after the initial head CT, with a significant drop in hematocrit secondary to hemothorax $(28.7 \%$ compared with $37.4 \%$ on admission), ultimately requiring intubation and chest tube placement. Subsequent repeat head CT demonstrated a temporal contusion and subarachnoid hemor- rhage, which was unchanged on subsequent head CT performed 6 hours later. This patient was managed nonoperatively and discharged after a 7-day hospitalization without further imaging.

\section{DISCUSSION}

Our findings demonstrate that isolated falcine and tentorial SDHs tend to be small (mean size, $2.8 \mathrm{~mm}$ thick) and do not increase on repeat CT examinations. To the authors' knowledge, this is the largest study to date to assess short-term repeat imaging in patients with isolated falcine or tentorial SDH.

Most published literature regarding isolated falcine and tentorial SDH is limited to case reports and series, ${ }^{10-13}$ except for 2 relatively recent retrospective studies. Sweis et $\mathrm{l}^{8}$ reported clinical features and outcomes of 27 patients with isolated falcine or tentorial SDH. They reported favorable clinical outcomes without short hospital stays and no need for surgical intervention. A more recent study by Howard et $\mathrm{al}^{9}$ reported both imaging and clinical outcomes of 65 patients with isolated falcine and tentorial SDH, noting no significant change in SDH size on repeat imaging. Present data build on these studies and further support the conclusion that repeat CT in patients with TBI with isolated falcine or tentorial SDH may not be necessary.

There are several limitations to the current study. First, the retrospective approach may subject the current analysis to inherent observation bias. Second, only 6 patients in the current analysis presented with a GCS score of $<13$, which may make the overall results less generalizable to this subgroup. Finally, the cohort studied includes only 16 patients receiving antiplatelet and/or anticoagulation medication, of whom 6 patients received anticoagulation with an average international normalized ratio of 2.9. Howard et $\mathrm{al}^{9}$ also found similar findings of lack of SDH expansion in 12 patients receiving antiplatelet and/or anticoagulation medication. Sweis et $\mathrm{al}^{8}$ reported no change in clinical outcomes in 3 patients with isolated falcotentorial SDH receiving warfarin anticoagulation. Additional studies with significantly larger numbers of anticoagulated patients are needed to more conclusively define the natural history of isolated falcine and tentorial SDH in patients with a history of anticoagulation or coagulopathy and to inform recommendations regarding repeat imaging in this subgroup of patients.

\section{CONCLUSIONS}

Our study suggests that most patients with blunt TBI, especially those with mild TBI presenting with isolated tentorial or falcine $\mathrm{SDH}$, may not require routine repeat head CT examinations in the absence of clinical deterioration. Repeat examinations in this group of patients may result in unnecessary cost and increased length of hospital stay. The decision to repeat imaging should be based on clinical suspicion of worsening hemorrhage, with repeat imaging being reserved for those patients with new focal neurologic deficits, acute changes in mental status, or other complications of polytrauma such as hypotension.

Disclosures: Geoff Manley-UNRELATED: Grant: National Institutes of Health and Department of Defense, Comments: research funding for traumatic brain injury studies.* Jason Talbott-UNRELATED: Consultancy: StemCells Inc, Comments: member of data-monitory committee for stem cell clinical trial. This study has 
concluded, and I am no longer involved; Expert Testimony: Tindall Bennett and Shoup. *Money paid to the institution.

\section{REFERENCES}

1. Wintermark M, Sanelli PC, Anzai Y, et al. Imaging evidence and recommendations for traumatic brain injury: advanced neuro- and neurovascular imaging techniques. AJNR Am J Neuroradiol 2015;36: E1-11 CrossRef Medline

2. Massaro F, Lanotte M, Faccani G, et al. One hundred and twentyseven cases of acute subdural haematoma operated on: correlation between CT scan findings and outcome. Acta Neurochir (Wien) 1996;138:185-91 CrossRef Medline

3. McKhann GM II, Copass MK, Winn RH. Prehospital care of the head-injured patient. In: Narayan RK, Wilberger JE, Povlishock JT, ed. Neurotrauma. New York: McGraw-Hill; 1996:103-18

4. Meyer SH, Chestnut M. Posttraumatic extra-axial mass lesions: subdural and extraudural hematoma. In: Tindall GT, Cooper PR, Barrow DL, eds. The Practice of Neurosurgery. Baltimore: Williams and Wilkins; 1996:1443-60

5. Bajsarowicz P, Prakash I, Lamroureux J, et al. Nonsurgical acute traumatic subdural hematoma: what is the risk? J Neurosurg 2015; 123:1176-83 CrossRef Medline

6. Karibe H, Hayashi T, Hirano T, et al. Surgical management of trau- matic acute subdural hematoma in adults: a review. Neurol Med Chir (Tokyo) 2014;54:887-94 CrossRef Medline

7. Bullock MR, Chesnut R, Ghajar J, et al; Surgical Management of Traumatic Brain Injury Author Group. Surgical management of acute subdural hematomas. Neurosurgery 2006;58:S16-24; discussion Si-iv Medline

8. Sweis RT, Ouyang B, Lopez GA, et al. Falcine and tentorial subdural hematomas may not routinely require transfer to a tertiary care center. J Emerg Med 2015;49:679-85 CrossRef Medline

9. Howard BM, Rindler R, Holland CM, et al. Management and outcomes of isolated tentorial and parafalcine "smear" subdural hematomas at a level-1 trauma center: necessity of high acuity care. J Neurotrauma 2017;34:128-36 CrossRef Medline

10. Bartels RH, Verhagen WI, Prick MR, et al. Interhemispheric subdural hematoma in adults: case reports and a review of the literature. Neurosurgery 1995;36:210-14 Medline

11. Takeuchi S, Takasato Y, Massoka H, et al. Traumatic peritentorial subdural hematomas: a study of 32 cases. Turk Neurosurg 2012;22: 305-08 CrossRef Medline

12. Want Y, Wang C, Cai S, et al. Surgical management of traumatic interhemispheric subdural hematoma. Turk Neurosurg 2014;24: 228-33 CrossRef Medline

13. Vaz R, Duarte F, Oliveria J, et al. Traumatic interhemispheric, subdural haematomas. Acta Neurochir 1991;111:128-31 CrossRef Medline 